\title{
Characterization of the thermalisation efficiency and photostability of photoacoustic contrast agents
}

\author{
T. Stahl, T. Allen, P. Beard \\ Department of Medical Physics and Bioengineering, University College London, Gower Street, \\ London WC1E 6BT, UK
}

\begin{abstract}
A simple method of characterizing organic dyes and nanoparticles used as contrast agents for photoacoustic molecular imaging based on relative photoacoustic measurements is described. By acquiring just two time-resolved photoacoustic signals, one in the sample of interest and the other in water, measurements of the thermalisation efficiency and other parameters relevant to the characterization of contrast agents can be acquired. The method was validated using absorbing solutions of known thermalisation efficiency and Grüneisen coefficient. It was then used to measure the thermalisation efficiency of solutions of gold nanorods, rhodamine B, methylene blue, IR-820, fluorescein and cresyl violet. In addition, photoacoustic measurements of the photostability of these substances were acquired.
\end{abstract}

Keywords: Photoacoustic efficiency, Contrast agents, Thermalisation efficiency, Photostability, Grüneisen coefficient

\section{INTRODUCTION}

Exogenous contrast agents in photoacoustic imaging[1] have been employed for the detection of cancer cells[2], sentinel lymph nodes[3], angiogenesis[4], and the characterization of atherosclerotic plaques[5]. However, organic dye based contrast agents can exhibit ground state bleaching, fluorescence and other processes that can diminish their efficiency as photoacoustic contrast agents. The photoacoustic signal generation efficiency of nanoparticle based contrast agents may also be substantially alternated by mismatching the thermodynamic properties of the particle compared to the surrounding medium. For this reason we have developed a simple method based on relative photoacoustic measurements that permits measurement of the conversion efficiency of (i) the absorbed optical energy to heat (the thermalisation efficiency) and (ii) heat energy to stress (the Grüneisen coefficient). Section 2 describes the underlying principles of the technique, section 3 the experimental method and in section 4 measurements of photoacoustic spectra, thermalisation efficiency and photostability of a number of different organic dye and nanoparticle based contrast agents are described.

\section{METHOD}

The photoacoustic effect describes the process in which absorbed electromagnetic energy is converted to an acoustic pressure wave. In practice, a short (usually nanoseconds in duration) light pulse is used to initiate an excited state at the excitation wavelength. The deposited light energy will dissipate via vibrational relaxation of the excited state, rapidly heating the surrounding medium. The rapid temperature rise causes a pressure increase and generates a broadband acoustic pressure wave which propagates away from the source. The initial pressure, $\mathrm{P}_{0}$, generated by a collimated light beam incident on a homogeneous, purely absorbing medium can be described by

$$
P_{0}=\Gamma E_{t} \mu_{a} \Phi_{0} e^{-\left(\mu_{a} \cdot z\right)}
$$

where $E_{t}$ is the thermalisation efficiency which represents the conversion efficiency of absorbed energy to heat. $\Gamma$ is the Grüneisen coefficient of the surrounding medium and describes the conversion of heat energy to stress, $\mu_{\mathrm{a}}$ is the absorption coefficient of the sample, $\Phi_{0}$ is the incident fluence and $\mathrm{z}$ is the axial distance from the surface of the absorber. The acoustic wave propagates towards the surface and is detected by an ultrasound transducer. Assuming negligible acoustic attenuation, the initial compressive part of the detected signal, $\mathrm{S}(\mathrm{t})$ can be expressed as

Photons Plus Ultrasound: Imaging and Sensing 2014, edited by Alexander A. Oraevsky, Lihong V. Wang, Proc. of SPIE Vol. 8943, 89435H · @ 2014 SPIE · CCC code: 1605-7422/14/\$18 · doi: 10.1117/12.2039694 


$$
S(t)=K \Gamma E_{t} \mu_{a} \Phi_{0} e^{-\left(\mu_{a} \cdot c_{s} \cdot\left(t-t_{0}\right)\right)}
$$

where $\mathrm{K}$ is a scaling factor that represents the detector sensitivity. $\mathrm{c}_{\mathrm{s}}$ is the speed of sound of the medium and $\mathrm{t}_{0}$ is the time at which the signal amplitude is a maximum. $\mu_{\mathrm{a}}$ can be obtained by fitting an exponential to the signal (for $\mathrm{t}<\mathrm{t}_{0}$ ) as illustrated in Figure 1.

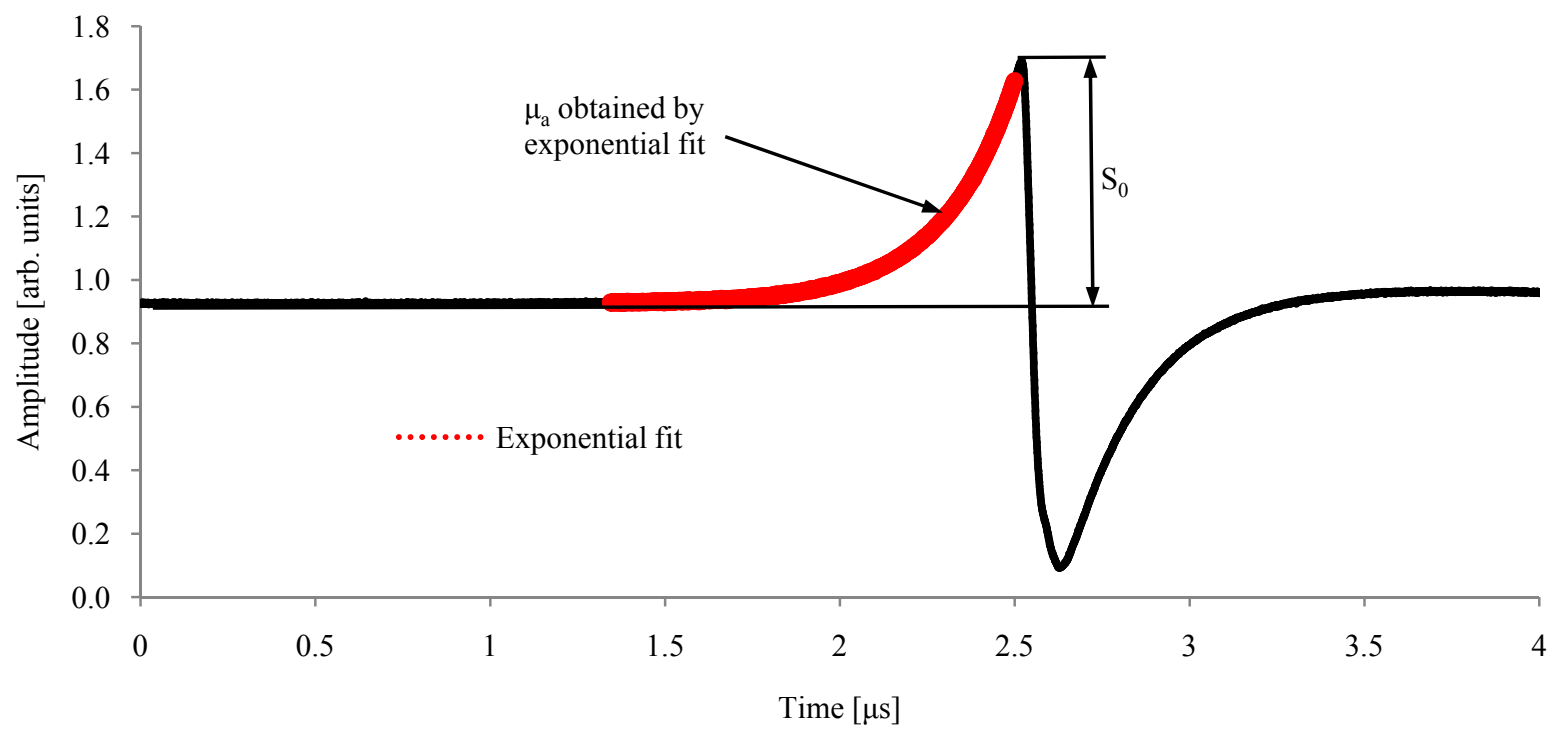

Figure 1: Example of photoacoustic signal generated by an absorber (solid, black line), its corresponding exponential fit (dashed, red line) for the determination of $\mu_{\mathrm{a}} \mathrm{S}_{0}$ is the peak positive amplitude at time $\mathrm{t}=\mathrm{t}_{0}$.

The method for estimating the $E_{t}$ of the sample is based upon comparison of the photoacoustic signal generated in the sample with that generated in a reference absorber of known $E_{t}$ and $\Gamma$. In this study, water was used as the reference absorber because it is known to exhibit only non-radiative relaxation so $E_{t}$ can be assumed to be 1 . In addition $\Gamma$ can be accurately calculated from the known thermodynamic properties of water.

For the sample, the signal peak amplitude $S_{0, \text { sample }}$ at $\mathrm{t}_{0}$ is given by,

$$
S_{0, \text { sample }}=K E_{t, \text { sample }} \Gamma_{\text {sample }} \mu_{a, \text { sample }} \Phi_{0}
$$

Assuming the reference photoacoustic signal is obtained using under identical experimental conditions so that $\Phi_{0}$ and $K$ are unchanged and that $\mathrm{E}_{\mathrm{t}}=1$, the signal amplitude at $\mathrm{t}_{0}$ for the reference absorber is:

$$
S_{0, \text { reference }}=K \Gamma_{\text {reference }} \mu_{a, \text { reference }} \Phi_{0} .
$$

The ratio of equation (3) and (4) is:

$$
\frac{S_{0, \text { sample }}}{S_{0, \text { reference }}}=\frac{E_{t, \text { sample }} \Gamma_{\text {sample }} \mu_{a, \text { sample }}}{\Gamma_{\text {reference }} \mu_{a, \text { reference }}}
$$

By rearranging $(5), E_{\mathrm{t}, \text { sample }}$ is given by:

$$
E_{\mathrm{t}, \text { sample }}=\frac{S_{0, \text { sample }} / \mu_{a, \text { sample }}}{S_{0, \text { reference }} / \mu_{\text {a,reference }}} \cdot \frac{\Gamma_{\text {reference }}}{\Gamma_{\text {sample }}}
$$


Equation 6 shows that by acquiring photoacoustic signals in both the sample and reference absorber, and recovering the peak amplitudes and absorption coefficients (the latter being obtained from the exponential fit to the signals), and knowing the ratio of their Grüneisen coefficients, it is possible to estimate the thermalisation efficiency of the sample.

In this study, the sample comprised an absorber in solution. In order to determine the Grüneisen coefficient of the solution $\left(\Gamma_{\text {sample }}\right)$, a photoacoustic signal was acquired at a wavelength $\lambda_{0}$ at which absorption by the absorber was negligible but significant in the solvent. A signal was then acquired under identical experimental conditions in water. Assuming the thermalisation efficiency of the solvent is $100 \%$, and with knowledge of the Grüneisen coefficient of water $\Gamma_{H 20}$, it is then possible to determine the $\Gamma_{\text {sample }}$ as follows:

$$
\Gamma_{\text {sample }}=\frac{S_{0, \text { sample }}\left(\lambda_{0}\right) / \mu_{a, \text { sample }}\left(\lambda_{0}\right)}{S_{0, \text { reference }}\left(\lambda_{0}\right) / \mu_{a, \text { reference }}\left(\lambda_{0}\right)} \Gamma_{H 20}
$$

\section{EXPERIMENTAL}

\subsection{Materials}

Copper $^{(\mathrm{II})}$ chloride, nickel ${ }^{(\mathrm{II})}$ chloride, rhodamine B, IR-820, methylene blue, fluorescein, cresyl violet, single walled carbon nanotubes (from here on referred to as carbon nanotubes), sodium hydroxide, methanol and ethanol were all purchased from Sigma Aldrich (UK). Gold nanorods (aspect ratio of 4.1; cetyltrimethyl-ammonium bromide ligand) were purchased from Nanopartz (USA). The solid substances were dissolved using methanol, ethanol, distilled water $(\sim 18 \mathrm{M} \Omega \mathrm{cm})$ or aqueous sodium hydroxide solution $(0.1 \mathrm{M})$ to make stock solutions, which were further diluted for the experiments. The carbon nanotubes were conjugated to polyethylene glucose in order to gain a homogenous solution of the particles in water.

\subsection{Experimental Setup}

The experimental setup used in this study is depicted in Figure 2. It consists of a Nd:YAG pumped optical parametric oscillator (OPO) laser, tunable from 400-2000nm, with a pulse lengths of 7ns (GWU, Spectra-Physics), coupled into a $1.5 \mathrm{~mm}$ inner diameter fused silica fiber. A glass-slide between laser output and fiber reflects light onto the opening of an integrating sphere containing a photodiode. The distal end of the fiber is clamped about $3 \mathrm{~cm}$ away from the surface of the absorbing sample solution. The fluence at the output of the fiber, illuminating the sample solution, varies with wavelength, ranging from $2-10 \mathrm{mJcm}^{-2}$. The sample solution is contained in a homemade cuvette, located about $1 \mathrm{~cm}$ above the ultrasound transducer in a water tank. The ultrasound transducer is composed of a square $(10 \times 10 \mathrm{~mm})$, thin $(50 \mu \mathrm{m})$ polyvinylidene fluoride film, cemented with UV-curing glue onto a Perspex backing. A reading of the output of a photodiode contained within the integrating sphere and the signal reading of the ultrasound transducer are captured simultaneously with an oscilloscope and saved onto a computer. In a subsequent calibration step, a power-meter was used to relate the photodiode readings to the pulse energy incident on the target. 


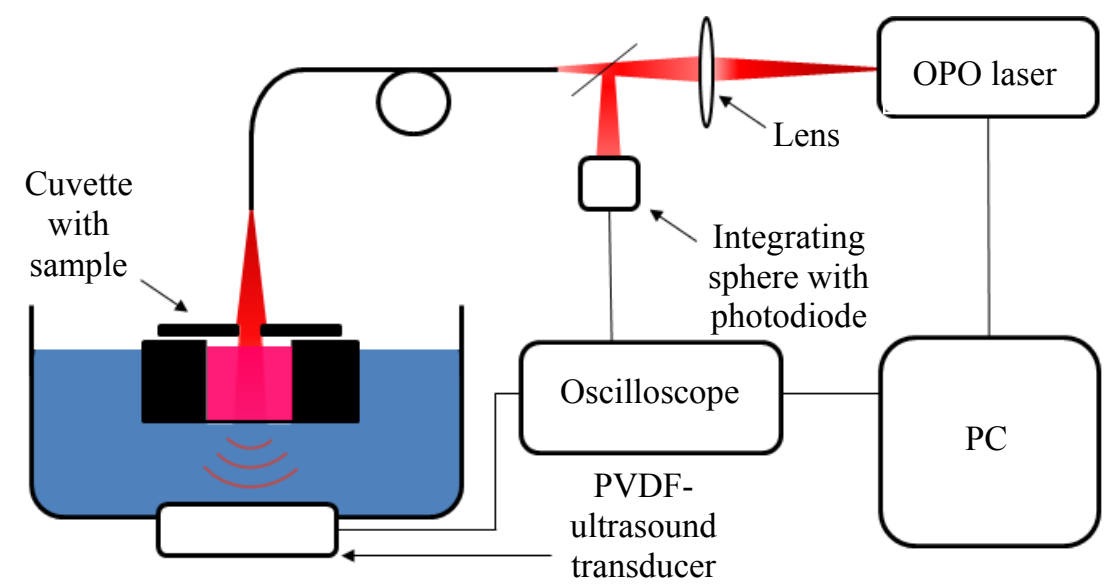

Figure 2: Experimental set-up

\subsection{Experimental validation}

To validate the method, measurements of absorbers of known thermalisation efficiency were made. In the first instance,

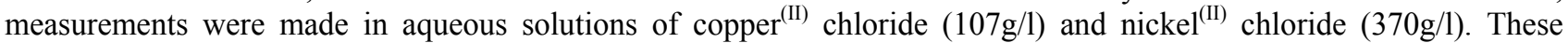
solutions were chosen because their thermalisation efficiencies can reasonably be assumed to be $100 \%$. In addition their Grüneisen coefficients are known to be significantly different to that of water[6] which provides an opportunity to test the ability of the method to correctly account for changes Grüneisen coefficients. In order to determine the Grüneisen coefficients using equation $7, \lambda_{0}$ was chosen to be $1450 \mathrm{~nm}$ because at this wavelength both salts exhibit negligible absorption while that of water is significant. For both copper ${ }^{(\mathrm{II})}$ chloride and nickel ${ }^{(\mathrm{II})}$ chloride, the values of $\mathrm{E}_{\mathrm{t}}$ obtained experimentally using the method described in section 2 were close to $100 \%$ as expected. In addition, a solution of rhodamine B in methanol $(400 \mu \mathrm{M})$ was evaluated since it exhibits significant radiative relaxation with a fluorescent quantum yield that is well known [6]. In this case, $\lambda_{0}$ was chosen to be $1550 \mathrm{~nm}$ due to the strong absorption of methanol at this wavelength compared to that of rhodamine B. The experimentally measured value of Et was found to be $40 \%$. This is consistent with the fluorescent quantum yield (QY) $(\sim 60 \%$ at $400 \mu \mathrm{M})$ reported in the literature[7] assuming $\mathrm{E}_{\mathrm{t}}=$ $1-\mathrm{QY}$.

In addition, as a further test, the Grüneisen coefficients of pure methanol and ethanol were estimated using equation 7 and compared to the values obtained using

$$
\Gamma=\frac{\beta c^{2}}{C_{p}}
$$

where $\beta$ is volume thermal expansion coefficient, $c$ is the sound speed and $\mathrm{C}_{\mathrm{p}}$ is the heat capacity at constant pressure. The measured and calculated values of the Grüneisen coefficients in close agreement: 0.44 and 0.48 respectively for methanol and 0.60 and 0.61 for ethanol.

\subsection{Measurement of thermalisation efficiency, photoacoustic spectra and photostability of various photoacoustic contrast agents}

The setup depicted in Figure 2 was utilized for the measurement of $\mathrm{E}_{\mathrm{t}}$, the photoacoustic spectra and the photostability of six contrast agents, namely: gold nanorods, carbon nanotubes, methylene blue, IR-820, cresyl violet and fluorescein. Gold nanorods, carbon nanotubes and methylene blue were dissolved in water, cresyl violet was dissolved in ethanol and fluorescein was dissolved in $0.1 \mathrm{M}$ sodium hydroxide. In order to measure $\mathrm{S}_{0}$ and $\mu_{\mathrm{a}}$ for the determination of $\mathrm{E}_{\mathrm{t}}$, each of the sample solutions were introduced into a cuvette individually and placed in the water tank as shown in Figure 2.

To obtain the photoacoustic spectra, measurements of $S_{0}$ and $\mu_{a}$ were obtained for each wavelength within the range required. The photostability measurements were performed using an enclosed cuvette to prevent solvent evaporation. For 
the measurement the sample was continuously irradiated for $30 \mathrm{~min}$ with pulsed laser light $(\mathrm{PRF}=10 \mathrm{~Hz})$ at the wavelength of the peak absorption of the sample whilst recording $\mathrm{S}_{0}$ once every minute.

\section{RESULTS}

\subsection{Photoacoustic spectra}

Photoacoustic spectra were recorded in order to determine the wavelength at which the samples have the highest absorption. The wavelength of peak absorption was chosen for the determination of $\mathrm{E}_{\mathrm{t}}$. The measured photoacoustic spectra of various absorbers, generated via plotting the measured $\mu_{\mathrm{a}}$ as a function of wavelength, are shown in Figure 3 . The $\mu_{\mathrm{a}}$ spectra plotted below are in good agreement with the photoacoustic amplitude spectra and the supplier specification sheets (data not shown in Figure 3).

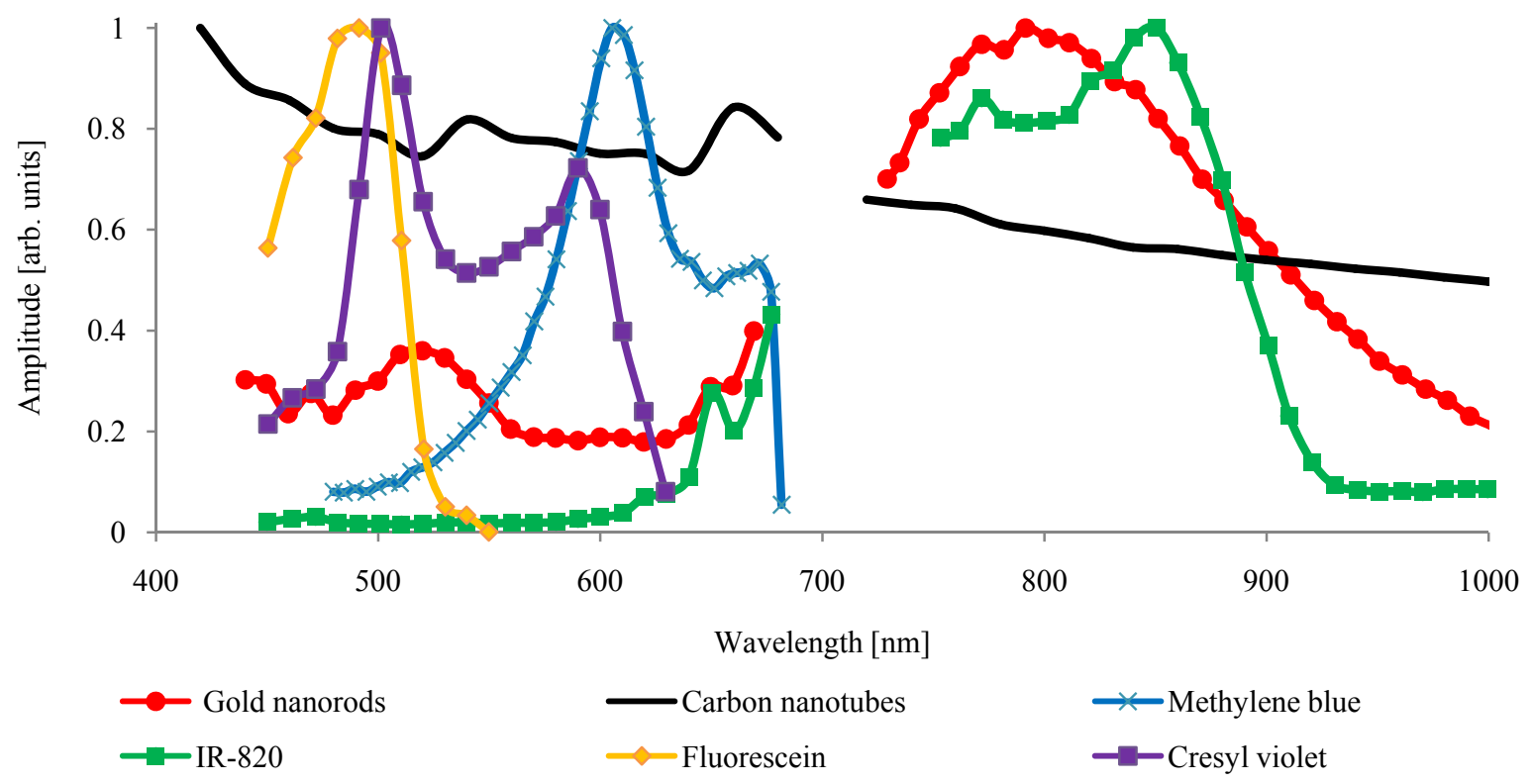

Figure 3: Measurements of the photoacoustic spectra of gold nanorods, carbon nanotubes, methylene blue, IR-820, cresyl violet and fluorescein. 


\subsection{Thermalisation efficiency}

The results of the $\mathrm{E}_{\mathrm{t}}$ measurements are shown below (Table 1). Due to the strong broadband absorption of the carbon nanotube sample throughout the whole spectrum covered by the OPO laser it wasn't possible to account for the Grüneisen coefficient and thus measure $E_{t}$. The values of $E_{t}$ of the other dyes included in this study, are in good agreement with their quantum yield values reported in the literature.

Table 1: Results of the $\mathrm{E}_{\mathrm{t}}$ measurements using the method proposed here and literature values for comparison.

\begin{tabular}{|c|c|c|c|c|}
\hline \multirow[t]{2}{*}{ Absorber } & \multicolumn{2}{|c|}{$\begin{array}{l}\text { Thermalisation efficiency } \\
\qquad\left(\mathbf{E}_{t}\right)\end{array}$} & \multirow{2}{*}{$\begin{array}{c}\text { Measured molar } \\
\text { extinction coefficient } \\
(\varepsilon) \\
{\left[\mathrm{cm}^{-1} \mathbf{M}^{-1}\right]}\end{array}$} & \multirow{2}{*}{$\begin{array}{c}\text { Peak absorption } \\
\text { wavelength } \\
{[\mathrm{nm}]}\end{array}$} \\
\hline & Measured & Literature & & \\
\hline Gold nanorods & $100 \%$ & $100 \%[8]$ & $2.0 \cdot 10^{9}$ & 820 \\
\hline Carbon nanotubes & - & $100 \%[9]$ & $2.7 \cdot 10^{5}$ & 450 \\
\hline IR-820 & $95 \%$ & $96 \%[10]$ & $1.5 \cdot 10^{5}$ & 820 \\
\hline Methylene blue & $100 \%$ & $98 \%[11]$ & $4.4 \cdot 10^{4}$ & 605 \\
\hline Cresyl violet & $47 \%$ & $51 \%[12]$ & $6.2 \cdot 10^{4}$ & 500 \\
\hline Fluorescein & $76 \%$ & $79 \%[13]$ & $8.1 \cdot 10^{4}$ & 490 \\
\hline
\end{tabular}

\subsection{Photostability}

Figure 4 shows the results of the photostability experiments. The plot shows the measured signal amplitude over the number of laser pulses the sample was irradiated with. During the measurement period, gold nanorods, carbon nanotubes and methylene blue were shown to be highly photostable. For the gold nanorods and the carbon nanotube sample this was expected, as they don't rely on the absorption of light via conjugated $\pi$-electron systems prone to dissociation[14]. On the contrary, the methylene blue sample was expected to show some photostability[15]. This unexpected behavior may be due to the relatively high concentration used in this particular experiment. IR-820, cresyl violet and fluorescein exhibit considerable reduction in the measured amplitude over exposure to laser pulses. Fluorescein has the strongest dependency with a loss in signal amplitude of about 30\% after 30min, whereas the signal amplitude of IR- 820 and cresyl violet is reduced by only 14 and $19 \%$, respectively. This is consistent with previous studies based upon purely optical measurements [16-18]. 


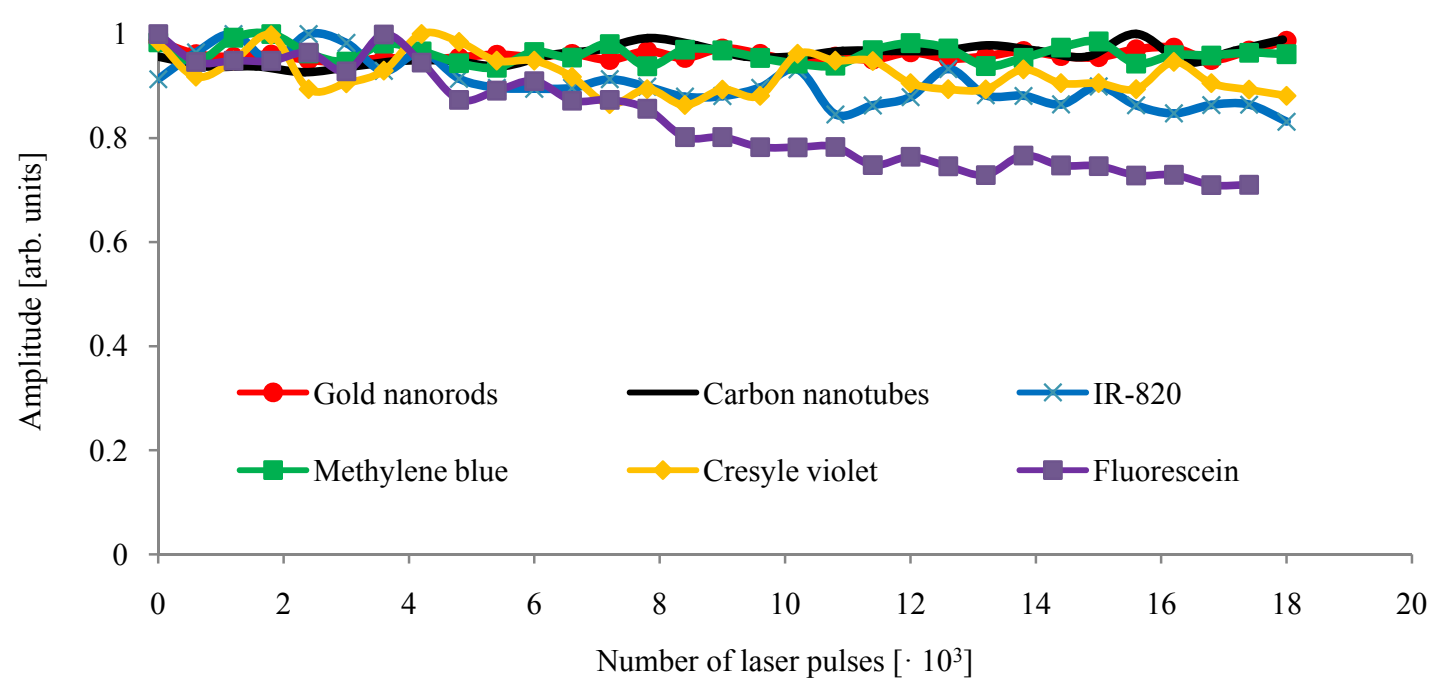

Figure 4: Measurements of photostability: photoaacoustic signal amplitude versus number of laser pulses.

\section{CONCLUSION}

We have successfully demonstrated the feasibility of a method for measuring the thermalisation efficiency of organic dyes and nanoparticles used as contrast agents in photoacoustic molecular imaging. The method has been validated using copper $^{(\mathrm{II})}$ chloride, nickel ${ }^{(\mathrm{II})}$ chloride and rhodamine B and applied to solutions of gold nanorods, methylene blue, IR820 , fluorescein and cresyl violet. In all the cases the measured $\mathrm{E}_{\mathrm{t}}$ agreed well with the literature values. The advantage of this approach is that it permits measurement of all the key parameters (thermalisation efficiency, Grüneisen coefficient and absorption coefficient) involved in photoacoustic signal generation by acquiring just two photoacoustic signals. It therefore represents a simple and rapid method of comprehensively evaluating photoacoustic contrast agents.

\section{REFERENCES}

[1] P. Beard, "Biomedical photoacoustic imaging," Interface Focus 1(4), 602-631 (2011) [doi:10.1098/rsfs.2011.0028].

[2] J. V Jokerst, A. J. Cole, D. Van de Sompel, and S. S. Gambhir, "Gold Nanorods for Ovarian Cancer Detection with Photoacoustic Imaging and Resection Guidance via Raman Imaging in Living Mice.," ACS nano 6(11), 10366-10377 (2012) [doi:10.1021/nn304347g].

[3] K. H. Song, C. Kim, C. M. Cobley, Y. Xia, and L. V Wang, "Near-infrared gold nanocages as a new class of tracers for photoacoustic sentinel lymph node mapping on a rat model.," Nano letters 9(1), 183-188 (2009) [doi:10.1021/nl802746w].

[4] D. Pan, M. Pramanik, A. Senpan, J. S. Allen, H. Zhang, S. a Wickline, L. V Wang, and G. M. Lanza, "Molecular photoacoustic imaging of angiogenesis with integrin-targeted gold nanobeacons.," FASEB journal 25(3), 875882 (2011) [doi:10.1096/fj.10-171728].

[5] B. Wang, P. Joshi, V. Sapozhnikova, J. Amirian, S. H. Litovsky, R. Smalling, K. Sokolov, and S. Emelianov, "Intravascular Photoacoustic Imaging of Macrophages using Molecular Targeted Gold Nanoparticles," Proc. SPIE 7564, A. A. Oraevsky and L. V. Wang, Eds., 75640A-75640A-7 (2010) [doi:10.1117/12.841070]. 
[6] J. Laufer, E. Zhang, and P. Beard, "Evaluation of Absorbing Chromophores Used in Tissue Phantoms for Quantitative Photoacoustic Spectroscopy and Imaging," IEEE Journal of Selected Topics in Quantum Electronics 16(3), 600-607 (2010) [doi:10.1109/JSTQE.2009.2032513].

[7] B. R, H. S, I. C, N. V, and Vallabhan C, "Pulsed photoacoustic determination of absolute quantum yield of the laser dye rhodamine b," Modern Physics Letters B 10(22), 1103-1110 (1996).

[8] C.-Z. Li, K. B. Male, S. Hrapovic, and J. H. T. Luong, "Fluorescence properties of gold nanorods and their application for DNA biosensing," Chemical communications 1(31), 3924-3926 (2005) [doi:10.1039/b504186d].

[9] M. J. O'Connell, S. M. Bachilo, C. B. Huffman, V. C. Moore, M. S. Strano, E. H. Haroz, K. L. Rialon, P. J. Boul, W. H. Noon, et al., "Band gap fluorescence from individual single-walled carbon nanotubes.," Science 297(5581), 593-596 (2002) [doi:10.1126/science.1072631].

[10] D. S. Conceição, D. P. Ferreira, and L. F. V. Ferreira, "Photochemistry and Cytotoxicity Evaluation of Heptamethinecyanine Near Infrared (NIR) Dyes.," International journal of molecular sciences 14(9), 1855718571 (2013) [doi:10.3390/ijms140918557].

[11] J. Olmsted, "Calorimetric Determinations of Absolute Fluorescence Quantum Yields," The Jounal of Physical Chemistry 83(20), 2581-2584 (1979).

[12] S. J. Isak and E. M. Eyring, "Fluorescence Quantum Yield of Cresyl Violet in Methanol and Water as a Function of Concentration," Journal of physical chemistry 58(15), 1738-1742 (1992).

[13] G. A. Crosby and J. N. Demas, “The measurement of Photoluminescence Quantum Yields," The Journal of Physical Chemistry 75(8), 991-1024 (1971).

[14] J.-W. Kim, E. I. Galanzha, E. V Shashkov, H.-M. Moon, and V. P. Zharov, "Golden carbon nanotubes as multimodal photoacoustic and photothermal high-contrast molecular agents.," Nature nanotechnology 4(10), 688-694, Nature Publishing Group (2009) [doi:10.1038/nnano.2009.231].

[15] C. K. Sheng and W. M. M. Yunus, "Study of Photobleaching Mechanism in Methylene Blue Sensitized Gelatin Using a Single Beam UV-Vis . Fibre Optics Spectrophotometer," J. Sci. \& Technol. 13(1), 23-30 (2005).

[16] A. Philip and P. Radhakrishnan, "Monitoring the photobleaching of cresyl violet in polyvinyl alcohol using the photoacoustic effect," Optical Engineering 33(6), 1993-1994 (2014).

[17] L. Song, E. J. Hennink, I. T. Young, and H. J. Tanke, "Photobleaching kinetics of fluorescein in quantitative fluorescence microscopy.," Biophysical journal 68(6), 2588-2600 (1995) [doi:10.1016/S0006-3495(95)80442$\mathrm{X}]$.

[18] S. Otsuka-yao-matsuo, T. Omata, S. Ueno, and M. Kita, "Photobleaching of Methylene Blue Aqueous Solution Sensitized by Composite Powders of Titanium Oxide with $\mathrm{SrTiO} 3$, BaTiO 3 , and CaTiO 3," 2124-2129 (2003). 